\title{
Serum concentrations of selected proinflammatory cytokines in children with alopecia areata
}

\author{
Katarzyna Tabara, Magdalena Kozłowska, Anna Jędrowiak, Wojciech Bienias, Andrzej Kaszuba \\ Department of Dermatology, Pediatric and Oncological Dermatology, Medical University of Lodz, Lodz, Poland \\ Adv Dermatol Allergol 2019; XXXVI (1): 63-69 \\ DOI: https://doi.org/10.5114/ada.2019.82826
}

\begin{abstract}
Introduction: Alopecia areata (AA) is considered an autoimmune disorder characterized by patchy loss of hair from the scalp and other body parts. Many patients develop the disease in childhood.

Aim: To answer the question whether abnormal production of some proinflammatory cytokines (IL-2, IL-6, IL-15, IL-17A and IFN- $\gamma$ ) in children with AA may facilitate the development or progression of the disease.

Material and methods: The study group consisted of 42 children with AA, the control group - 37 healthy children. Peripheral venous blood samples were collected from patients with $A A$ and healthy controls and the concentrations of serum cytokines, namely IL-2, IL-6, IL-15, IL-17A, IFN- $\gamma$ were determined quantitatively by ELISA method.

Results: The serum IL-6, IL-15, IL-17A and IFN- $\gamma$ levels were significantly increased in patients with AA compared with control subjects $(p<0.05)$. The serum IL-15 level was found to be increased when the total duration of AA was increased ( $q=0.30 ; p=0.05$ ). The serum cytokine level of IL-17A was found to be decreased when duration of the current episode was longer than 2 years $(p<0.05)$, but the correlation between IL-17A serum level and duration of the current episode was not confirmed in the Spearman test $(q=-0.06 ; p=0.68)$. The serum IL-17A level was found to be significantly decreased when the thyroiditis was present $(q=-2.378 ; p<0.05)$.

Conclusions: The increased levels of serum IL-6, IL-15, IL-17A and IFN- $\gamma$ in children suggest imbalance in the serum proinflammatory cytokines production in AA.
\end{abstract}

Key words: alopecia, alopecia areata, cytokines, interleukins, children.

\section{Introduction}

Alopecia areata $(A A)$ is one of the most common types of hair loss in humans. Alopecia areata is considered an autoimmune disorder characterized by patchy loss of hair from the scalp and other body parts. Onset of AA may occur at any age; however, the majority of patients (50-60\%) develop the disease before the age of 20 years [1]. For this reason the disease is relatively common in children.

The etiology of AA is still not fully understood. About $20 \%$ of people with AA have a family history of the disease indicating a genetic predisposition [2]. Associations have been reported with a variety of genes, including major histocompatibility complex and cytokine genes, suggesting that the genetic predisposition is multifactorial in nature [3]. Physiological and emotional stresses, virus infections and trauma are triggers of AA [4].

A 'swarm of bees' is a characteristic histopathological feature in the lesions of AA [5]. However, the molecular mechanisms underlying this cell accumulation have not been uncovered. It has been proposed that the hair follicle is an immunologically 'privileged tissue' which is sheltered from immune surveillance by autoreactive T cells, and that failure of such immune privilege plays a key role in the pathogenesis of AA [6-8]. It is also suggested that hair follicle autoantigens, such as melanin-associated protein, may be recognized by cytotoxic T cells [4]. The association between AA and other autoimmune diseases suggests that AA itself is an autoimmune disease. Most evidence supports the role of altered T cell-mediated immunity in the pathogenesis of AA. Cytokines and chemokines play an important role in the immune process of the disease [4, 9-12].

Many authors draw attention to the fact that there is an incorrect level of some cytokines in the groups of patients with AA [4, 9-12]. However, to the best of our knowledge, there was only few such trials in the pediatric population [13].

Address for correspondence: Prof. Andrzej Kaszuba MD, PhD, Department of Dermatology, Pediatric and Oncological Dermatology, Medical University of Lodz, 1/5 Kniaziewicza St, 91-347 Lodz, Poland, phone: +48 42651 10 72, e-mail: andrzej.kaszuba@umed.lodz.pl Received: 23.10.2017, accepted: 26.11.2017. 


\section{Aim}

The aim of the study was to answer the question whether abnormal production of some proinflammatory cytokines (interleukin (IL)-2, IL-6, IL-15, IL-17A and interferon (IFN)- $\gamma$ ) in children with AA may facilitate the development or progression of the disease.

\section{Material and methods}

\section{Patient and control groups}

The study group consisted of 42 children with AA, below 18 years of age, hospitalized in the Department of Dermatology, Pediatric and Oncological Dermatology from March 2015 to September 2016. The control group consisted of 37 healthy children. The patient group in cluded 29 (69.1\%) female and 13 (30.9\%) male patients and the control group included 20 (54.1\%) female and 17 (45.9\%) male subjects. The mean ages of the patients with $\mathrm{AA}$ and the controls were $10.31 \pm 5.02$ years and $11.3 \pm 4.65$ years, respectively. Age, sex, duration of disease, site and age of onset, total number of patches, progression of disease, family history of AA, any systemic or autoimmune diseases and nail changes were recorded following the "Investigative guidelines for alopecia areata" [14]. Scalp involvement was assessed using the Severity of Alopecia Tool (SALT) [13]. The patients were classified into the following groups: (1a) AA patients with < 25\% scalp involvement; (1b) AA patients with 25-99\% scalp involvement; (2) patients with alopecia totalis (AT) or alopecia universalis (AU); (3) patients with ophiasis. In the present study, 15 patients were classified as (1a), 13 patients as (1b), 8 patients as (2) and 6 patients as (3). Eighteen (43\%) patients had nail involvement in the form of discoloration, nail pitting, frayed eponychium or longitudinal striations. Ten $(24 \%)$ patients had a family history of AA. There were 17 (40\%) patients with AA with a history of atopy (atopic dermatitis, allergic asthma or allergic rhinoconjunctivitis). There were 10 (24\%) patients in the examined group with thyroiditis and 2 (5\%) with vitiligo.

In the present study we recruited patients with AA undergoing an episode of attacks, and all patients had active disease. The patients who underwent either systemic immunosuppressant therapy or local treatment with glucocorticoids or dithranol before hospitalization were not included into the study. Also, children with signs of infection like fever, sore throat, cough or dysuria were excluded from the study. Investigations including blood tests (morphology, CRP, TSH, fT3, fT4, aTPO, aTG, IgE total, ANA) and trichoscopy were also performed to verify the inclusion criteria.

The total duration of the disease and duration of the current episode was defined as: (I) less than 3 months, (II) 3-12 months, (III) 12-24 months, (IV) 2-5 years, (V) more than 5 years.

Written informed consent from the parents of children with AA and control subjects was obtained. The study was carried out according to the Declaration of Helsinki Principles and was reviewed and approved by the Bioethics Committee of the Medical University of Lodz, Poland (RNN/191/14/KB).

\section{Methods}

Peripheral venous blood samples were collected from patients with AA and healthy controls. Sera were isolated and the concentrations of serum cytokines, namely IL-2, IL-6, IL-17A, IFN- $\gamma$ (Diaclone, Besancon Cedex, France) and IL-15 (R\&D Systems, Minneapolis, USA), were determined quantitatively in freshly collected serum samples by the enzyme-linked immunosorbent assay (ELISA) method in both patients and controls. ELISA tests were performed according to the manufacturer's instructions. The serum cytokine levels were compared between both groups and among the AA subtypes. The correlation of serum cytokine levels with sex, duration and extension of $A A$, nail involvement, associated autoimmune disorders, atopy and family history of AA was studied.

\section{Statistical analysis}

The Shapiro-Wilk test was used to determine the distribution of quantitative variables. The Mann-Whitney $U$-test was used to compare the median serum cytokine levels between the AA and control groups. The nonparametric test, Kruskal-Wallis ANOVA variance with the Dunn multiple post-hoc comparative test was used to compare median serum cytokine levels between the AA subgroups. The Spearman correlation test was used to study the correlation between quantitative parameters. The significance level was set at $p \leq 0.05$. Statistical analyses were performed using a software package Statistica v.12.

\section{Results}

The serum IL-6, IL-15, IL-17A and IFN- $\gamma$ levels were significantly increased in patients with AA compared with control subjects (Figure 1). In the analysis of cytokine concentrations in the subgroups according to sex, IL-6, IL-15, IL-17A and IFN- $\gamma$ levels were increased among the females, while in the male group, IL-15, IL-17A and IFN- $\gamma$ were increased but IL- 6 level was comparable between the AA and control group. The serum IL-2 level was slightly increased in patients with AA. Although, the difference between AA patients and the control group was just below the level of significance $(p=0.09)$ (Figure 2).

No significant difference was observed between the serum cytokine levels of IL-2, IL-6, IL-15, IL-17A and IFN- $\gamma$ when compared between the AA subgroups (Table 1). In addition, no correlation was found between serum cytokine levels and presence of nail involvement.

The serum IL-15 level was found to be increased when the total duration of AA was increased (Spearman correlation, $q=0.30 ; p=0.05)$. There was no correlation 

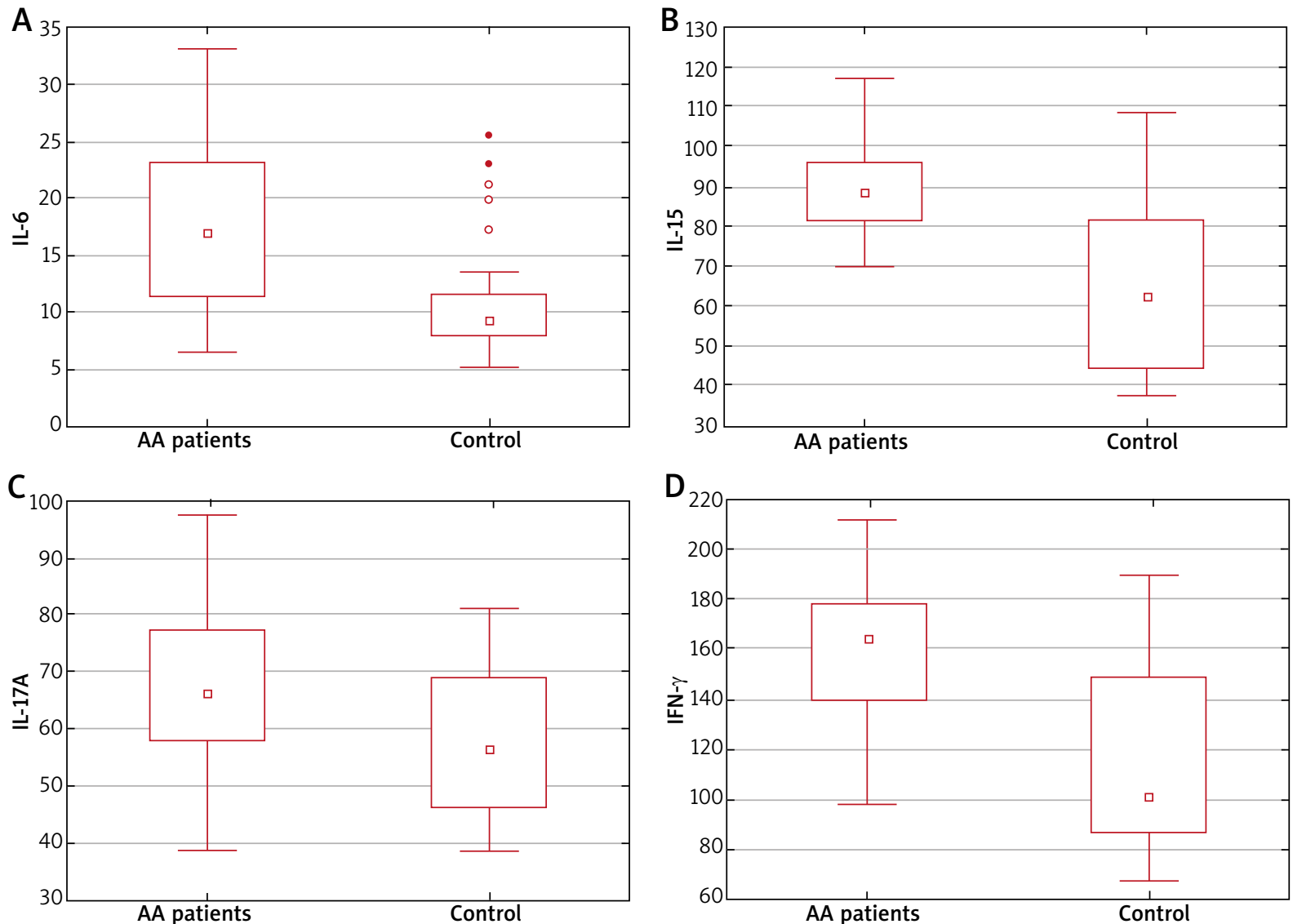

Figure 1. Significant difference in median plasma levels of IL-6 (A), IL-15 (B), IL-17A (C), IFN- $\gamma$ (D) between the study group (AA patients) and healthy controls

between other tested cytokines (IL-2, IL-6 and IFN- $\gamma$ ) and total duration of AA. Furthermore, the serum cytokine level of IL-17A was found to be decreased when duration of the current episode was longer than 2 years (ANOVA Kruskal-Wallis, $p<0.05$ ), but the correlation between IL-17A serum level and duration of the current episode was not confirmed in the Spearman test $(q=-0.06$; $p=0.68)$.

No correlation was found between serum cytokine levels and family history of AA.

The serum IL-17A level was found to be significantly decreased when the thyroiditis was present (Mann-Whitney, $q=-2.38$; $p<0.05)$. There was no correlation between other tested cytokines (IL-2, IL-6, IL-15 and IFN- $\gamma$ ) and presence of thyroiditis.

Finally, we found no significant difference in serum cytokine levels between patients with AA with and without atopy.

\section{Discussion}

Cytokines are a category of small proteins that are important in cell signaling. Cytokines are produced by

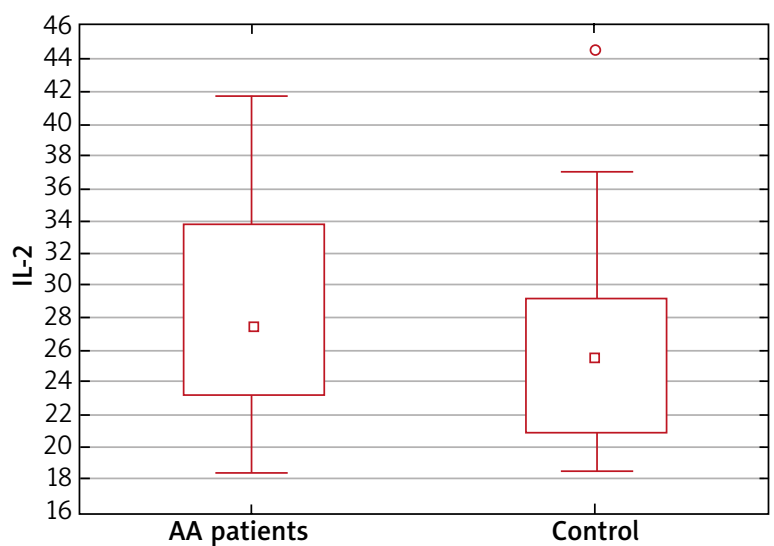

Figure 2. Non-significant difference in median plasma levels of IL-2 between the study group (AA patients) and healthy controls

a broad range of cells, including immune cells like macrophages, B lymphocytes, T lymphocytes and mast cells, as well as endothelial cells, fibroblasts, and various stromal cells. Cytokines modulate the balance between humoral and cell-based immune responses, and they regulate the 
Table 1. Comparison of the mean serum cytokine levels $(\mathrm{pg} / \mathrm{ml})$ in patients with alopecia areata (AA) in following groups: (1a) AA patients with < 25\% scalp ivolvement; (1b) AA patients with $25-99 \%$ scalp involvement; (2) patients with alopecia totalis (AT) or alopecia universalis (AU); (3) patients with ophiasis; (Total) whole group

\begin{tabular}{lccccc}
\hline Cytokine & $\begin{array}{c}\text { Mean value } \\
\text { (minimum-maximum) } \\
\text { in group (1a) }\end{array}$ & $\begin{array}{c}\text { Mean value } \\
\text { (minimum-maximum) } \\
\text { in group (1b) }\end{array}$ & $\begin{array}{c}\text { Mean value } \\
\text { (minimum-maximum) } \\
\text { in group (2) }\end{array}$ & $\begin{array}{c}\text { Mean value } \\
\text { (minimum-maximum) } \\
\text { in group (3) }\end{array}$ & $\begin{array}{c}\text { Mean value } \\
\text { (minimum-maximum) } \\
\text { in the whole group (Total) }\end{array}$ \\
\hline IL-2 & $27.82(19.06-37.10)$ & $26.41(18.45-37.10)$ & $30.94(19.81-41.70)$ & $28.76(20.18-38.80)$ & $28.22(18.45-41.70)$ \\
\hline IL-6 & $17.69(6.88-28.81)$ & $14.71(7.80-25.65)$ & $18.97(6.44-30.11)$ & $21.47(8.91-33.20)$ & $17.49(6.44-33.20)$ \\
\hline IL-15 & $87.33(69.85-108.60)$ & $86.93(69.80-102.64)$ & $91.27(69.70-102.34)$ & $99.19(79.90-117.43)$ & $89.69(69.70-117.43)$ \\
\hline IL-17A & $65.45(38.80-91.44)$ & $66.48(47.38-78.80)$ & $67.69(43.45-97.20)$ & $69.60(49.34-81.87)$ & $66.60(38.80-97.20)$ \\
\hline IFN- $\gamma$ & $153.97(97.88-184.80)$ & $160.19(131.20-189.90)$ & $169.30(127.81-204.43)$ & $155.10(101.80-210.80)$ & $158.70(97.88-210.80)$ \\
\hline
\end{tabular}

maturation, growth, and responsiveness of particular cell populations. Alteration in the specialized subpopulation of CD4+ T cells, namely T-helper (Th)1, Th2 and Th17 cells and regulatory $T$ cells (Tregs), has been implicated in autoimmune disorders [15-17].

AA is thought to arise when the hair follicle's (HF) natural immune privilege collapses, inducing ectopic MHC class I expression in the HF epithelium and autoantigen presentation to autoreactive CD8+ T cells. A 'swarm of bees' is a characteristic histopathological feature in the lesions of AA. However, the molecular mechanisms underlying this cell accumulation have not been uncovered. To attract immune cells around the hair bulb, some chemokines are upregulated in the hair bulbs. As in other autoimmune diseases, upregulation of proinflammatory cytokines must be critically implicated in AA pathogenesis. Evidence suggests that T cells and their effector cytokines play an important role in the selective loss of hair in AA [18-20]. The role of proinflammatory cytokines in the peripheral blood has been studied in AA, but mainly in the adult population. The purpose of this study was to elucidate the role of major proinflammatory cytokines, which has been suggested to be involved in AA pathogenesis previously, in children with AA.

The Th1 cytokine IL-2 is a lymphokine produced by activated T cells, and IL-2 receptor (IL-2R) is expressed on $T$ cells. The role of IL-2/IL-2R is implicated in the pathogenesis of AA [21, 22]. Redler et al. [23] investigated the association between AA and selected cytokine genes using a sample of patients with AA and controls of Central European origin. A significant association was found for two variants of genes within both IL2RA and TNF/LTA. A phenocopy of human AA is induced in a mouse model by injecting IL-2-stimulated peripheral blood mononuclear cells intradermally to healthy human scalp skin xenotransplanted onto severe combined immunodeficiency mice [24]. Interestingly, Castela et al. [25] reported the use of low-dose IL-2 for treating severe AA by promoting the recruitment of Treg cells. The partial regrowth was achieved in 4 of 5 patients. In the present study, the serum IL-2 level was slightly increased in patients with AA. Although, the difference between AA patients and the control group was just below the level of significance. Valsecchi et al. [26] detected significantly higher concentrations of soluble IL-2R in patients in the active phase of AA compared with those in the stable phase, and in controls. Barahmani et al. [27] found higher serum IL-2 levels in patients with AA. Teraki et al. [28] reported significantly elevated serum levels of IL-2 in patients with the extensive form of AA compared with those with localized AA. Tembhre and Sharma [29] also reported higher levels of serum IL-2 in patients with active AA compared with the control group. In addition, a significant difference was found in serum IL-2 levels when compared among the AA subgroups, and a positive correlation was found between serum IL-2 levels and total duration of AA. To the best of our knowledge, up to now only one more study was made to examine the IL-2 level in the pediatric population, in which elevation of the soluble IL-2R level was also confirmed [30].

Interleukin-15 is a cytokine with structural similarity to IL-2. Interleukin-15 is secreted by mononuclear phagocytes and it regulates the activation and proliferation of CD8 memory T and natural killer (NK) cells [31]. However, IL-15 is an inflammatory cytokine involved in immunological memory including that to self-antigens, thereby playing a role in autoimmune diseases. These insights provide the scientific basis for clinical strategies directed toward diminishing IL-15 action. The monoclonal antibody Hu-Mik- $\beta 1$ targets the cytokine receptor subunit IL-2/IL-15R $\beta$, blocks IL-15 trans-presentation, and is being used in clinical trials in patients with autoimmune diseases [32]. In the present study the serum IL-15 level was significantly increased in children with AA compared with control subjects. The serum IL-15 level was found to be increased when the total duration of AA was increased. Upregulation of IL-15 and its receptor subunit IL-15R $\alpha$ in AA hair follicles were also described by Xing et al. [33] and Fuentes-Duculan et al. [34]. To the best of our knowledge, the present study is the only one that has demonstrated higher serum IL-15 in children with AA.

Interferon- $\gamma$ is an important activator of macrophages and inducer of class II major histocompatibility complex 
molecule expression. Aberrant IFN- $\gamma$ expression is associated with a number of autoinflammatory and autoimmune diseases. Interferon- $\gamma$ is produced predominantly by natural killer and natural killer T cells as part of the innate immune response, and by CD4 Th1 and CD8 cytotoxic $T$ lymphocyte effector $T$ cells once antigen-specific immunity develops. Interferen- $\gamma$ interferes with anagen hair growth [35]. This notion is supported by the present study, which found significantly higher circulating levels of IFN- $\gamma$ in children with AA compared with the control group. Tembhre et al. [29] also reported a higher serum IFN- $\gamma$ level in patients with active AA. A previous study also suggested higher serum levels of IFN- $\gamma$ in patients with AT or AU compared with controls, but no significant difference was found in levels of IFN- $\gamma$ between patients with localized AA and those with more extensive forms $[28,36]$. Contrary to this, levels of IFN- $\gamma$ mRNA were found to be lower in patients with AA than in healthy controls [37], and they were comparable in both the AA and control groups [27]. Shohat et al. [30] also found no statistically significant difference in the level of IFN- $\gamma$ in children with AA and the control group. Skurkovich et al. [38] administered anti-IFN- $\gamma$ intramuscularly to patients with AA. Anti-IFN- $\gamma$ antibodies were produced by immunizing goats with recombinant human IFN- $\gamma$, isolating the IgG, and preparing $F\left(a b^{\prime}\right) 2$ fragments. Patients with patchy AA had almost no recurrence with the significant improvement in hair growth, but patients with AU or AT had very limited terminal hair growth.

Interleukin 17 is a pro-inflammatory cytokine produced by Th17 cells and is induced by IL-23. The role of Th17 cells has been illustrated in the pathogenesis of many autoimmune disorders in human and animal models [39-41]. Aytekin et al. [42] found that the IL17 GG genotype was associated with susceptibility for AA, but this genotype was only present in a small number of patients. In the present study the serum IL-17A level was significantly increased in children with AA compared with control subjects. Furthermore, the serum cytokine level of IL-17A was found to be decreased when duration of the current episode was longer than 2 years, but the correlation between IL-17A serum level and duration of the current episode was not confirmed in the Spearman test. Interestingly, the serum IL-17A level was found to be significantly decreased when the thyroiditis was present. Atwa et al. [43] also described elevation of the serum concentration of IL-17 in patients with AA as compared with healthy controls. Tembhre et al. [29] reported higher serum IL-17A levels in patients with active AA, and IL-17A levels were also found to be varied among AA phenotypes, but the difference was of borderline significance. El-Morsy et al. [44] found that serum IL-17A was significantly higher in patients aged $\leq 30$ years than in patients aged $>30$ years. There was a significant negative correlation between age at disease onset and serum IL-17A. The data suggest that IL-17A may play a role in the induction or active phase of AA, indicating the involvement of
IL-17A and hence Th17 cells in facilitating the selective damage to hair follicles in AA [29].

Interleukin 6 is an interleukin that acts as both a proinflammatory cytokine and an anti-inflammatory myokine. Interleukin 6 is secreted by T cells (predominantly by Th17) and macrophages. In the present study, the serum IL-6 level in children was significantly increased in patients with AA compared with control subjects. In the analysis of cytokine concentrations in the subgroups according to sex, IL- 6 was increased among the females, while in the male group, the IL- 6 level was comparable between the AA and control group. Atwa et al. [43] reported a significantly higher IL-6 level in patients with AA as compared with healthy controls. Bilgic et al. [45] showed that the mean serum level of IL- 6 in AA patients was significantly higher than in the controls. However, in logistic regression analysis there was no relationship with the presence of AA. Shohat et al. [30] reported that the level of IL- 6 was significantly higher in the pediatric AA patients than the controls.

\section{Conclusions}

The study of T-cell subsets and associated cytokines is essential to develop a better understanding of the autoimmune nature of AA. However, circulating levels of cytokines could be affected by several factors, and these operate in complex networks. The current study is one of the few conducted among children with AA. In this study we highlight elevation of the serum proinflammatory cytokines IL-6, IL-15, IL-17A and IFN- $\gamma$ levels in children with AA compared with control subjects. Nowadays, none of the currently available therapeutic methods of AA bring long-term benefits. The spontaneous remission occurs in many cases of AA, but the disease can have a devastating impact on the patient quality of life. An elegant way to attenuate AA by simultaneous inhibition of IFN- $\gamma$, IL-2 and IL-15 signaling is the use of JAK inhibitors that act upstream of STATs. Although, further research is needed to confirm the effectiveness of JAK inhibitors therapy in patients with AA [46]. Further studies of comparative levels of serum and tissue cytokines in children are also required. These cytokines can be targeted to develop therapeutics and can be combined with current treatment regimens for better management of $A A$.

\section{Acknowledgments}

Statutory grant (number 502-03/5-064-01/502-54172) was awarded by the Medical University of Lodz, Poland.

\section{Conflict of interest}

The authors declare no conflict of interest. 


\section{References}

1. Garg S, Messenger AG. Alopecia areata: evidence-based treatments. Semin Cutan Med Surg 2009; 28: 15-8.

2. McDonagh AJG, Messenger AG. The pathogenesis of alopecia areata. Dermatol Clin 1996; 14: 661-70.

3. Petukhova L, Duvic M, Hordinsky M, et al. Genome-wide association study in alopecia areata implicates both innate and adaptive immunity. Nature 2010; 466: 113-7.

4. Ito T, Tokura Y. The role of cytokines and chemokines in the T-cell-mediated autoimmune process in alopecia areata. Exp Dermatol 2014; 23: 787-91.

5. Weedon D. Diseases of cutaneous appendages. In: Weedon's Skin Pathology. Weedon D (ed.). Churchill Livingstone, London 2002; 397-440.

6. Paus R, Ito N, Takigawa M, et al. The hair follicle and immune privilege. J Investig Dermatol Symp Proc 2003; 8: 188-94.

7. Kang H, Wu WY, Lo BK, et al. Hair follicles from alopecia areata patients exhibit alterations in immune privilegeassociated gene expression in advance of hair loss. J Invest Dermatol 2010; 130: 2677-80.

8. Messenger AG, McKillop J, Farrant P, et al. British Association of Dermatologists' guidelines for the management of alopecia areata 2012. Br J Dermatol 2012; 166: 916-26.

9. Gilhar A, Schrum AG, Etzioni A, et al. Alopecia areata: animal models illuminate autoimmune pathogenesis and novel immunotherapeutic strategies. Autoimmun Rev 2016; 15: 726-35.

10. McElwee KJ, Gilhar A, Tobin DJ, et al. What causes alopecia areata? Exp Dermatol 2013; 22: 609-26.

11. Ito T. Recent advances in the pathogenesis of autoimmune hair loss disease alopecia areata. Clin Dev Immunol 2013; 2013: 348546.

12. Brzezińska-Wcisło L, Bergler-Czop B, Wcisło-Dziadecka D, et al. New aspects of the treatment of alopecia areata. Postep Dermatol Alergol 2014; 31: 262-5.

13. Shohat M, Mimouni D, Ben-Amitai D. In vitro cytokine profile in childhood alopecia areata and the immunomodulatory effects of AS-101. Clin Exp Dermatol 2005; 30: 432-4.

14. Olsen EA. Investigative guidelines for alopecia areata. Dermatol Ther 2011; 24: 311-9.

15. Afzali B, Lombardi G, Lechler RI, et al. The role of T helper 17 (Th17) and regulatory T cells (Treg) in human organ trans plantation and autoimmune disease. Clin Exp Immunol 2007; 148: 32-46.

16. Furuzawa-Carlalleda J, Vargas-Rojas MI, Cabral AR. Autoimmune inflammation from the Th17 perspective. Autoimmun Rev 2007; 6: 169-75.

17. Weaver CT, Harrington LE, Mangan PR, et al. Th17: an effector CD4 T cell lineage with regulatory $T$ cell ties. Immunity 2006; 24: 677-88.

18. Kalish RS, Gilhar A. Alopecia areata: autoimmunity - the evidence is compelling. J Investig Dermatol Symp Proc 2003; 8: 164-7.

19. Ito T, Meyer KC, Ito $\mathrm{N}$, et al. Immune privilege and the skin. Curr Dir Autoimmun 2008; 10: 27-52.

20. King LE Jr, McElwee KJ, Sundberg JP. Alopecia areata. Curr Dir Autoimmun 2008; 10: 280-312.

21. Cantrell DA, Smith KA. The interleukin-2 T-cell system: a new cell growth model. Science 1984; 224: 1312-6.

22. Malek TR. The biology of interleukin-2. Annu Rev Immunol 2008; 26: 453-79.

23. Redler S, Albert F, Brockschmidt FF, et al. Investigation of selected cytokine genes suggests that IL2RA and the TNF/ LTA locus are risk factors for severe alopecia areata. Br J Dermatol 2012; 167: 1360-5.
24. Gilhar A, Schrum AG, Etzioni A, et al. Alopecia areata: animal models illuminate autoimmune pathogenesis and novel immunotherapeutic strategies. Autoimmun Rev 2016; 15: 726-35.

25. Castela E, Le Duff F, Butori C, et al. Effects of low-dose recombinant interleukin 2 to promote T-regulatory cells in alopecia areata. JAMA Dermatol 2014; 150: 748-51.

26. Valsecchi R, Imberti G, Martino D, Cainelli T. Alopecia areata and interleukin-2 receptor. Dermatology 1992; 184: 126-8.

27. Barahmani N, Lopez A, Babu D, et al. Serum T helper 1 cytokine levels are greater in patients with alopecia areata regardless of severity or atopy. Clin Exp Dermatol 2010; 35 : 409-16.

28. Teraki Y, Imanishi K, Shiohara T. Cytokines in alopecia areata: contrasting cytokine profiles in localized form and extensive form (alopecia universalis). Acta Derm Venereol 1996; 76 : 421-3.

29. Tembhre MK, Sharma VK. T-helper and regulatory T-cell cytokines in the peripheral blood of patients with active alopecia areata. Br J Dermatol 2013; 169: 543-8.

30. Shohat M, Mimouni D, Ben-Amitai D, et al. In vitro cytokine profile in childhood alopecia areata and the immunomodulatory effects of AS-101. Clin Exp Dermatol 2005; 30: 432-4.

31. Waldmann TA, Lugli E, Roederer M, et al. Safety (toxicity), pharmacokinetics, immunogenicity and impact on elements of the normal immune system of recombinant human IL-15 in rhesus macaques. Blood 2011; 117: 4787-95.

32. Waldmann TA. The biology of IL-15: implications for cancer therapy and the treatment of autoimmune disorders. J Investig Dermatol Symp Proc 2013; 16: 28-30.

33. Xing L, Dai Z, Jabbari A, et al. Alopecia areata is driven by cytotoxic T lymphocytes and is reversed by JAK inhibition. Nat Med 2014; 20: 1043-9.

34. Fuentes-Duculan J, Gulati N, Bonifacio KM, et al. Biomarkers of alopecia areata disease activity and response to corticosteroid treatment. Exp Dermatol 2016; 25: 282-6.

35. Sato-Kawamura M, Aiba S, Tagami H. Strong expression of CD40, CD54 and HLA-DR antigen and lack of evidence for direct cellular cytotoxicity are unique immunohistopathological features in alopecia areata. Arch Dermatol Res 2003; 12: $536-43$

36. Arca E, Musabak U, Akar A, et al. Interferon-gamma in alopecia areata. Eur J Dermatol 2004; 14: 33-6.

37. Katagiri K, Arakawa S, Hatano Y. In vivo levels of IL-4, IL-10, TGFbeta1 and IFN-gamma mRNA of the peripheral blood mononuclear cells in patients with alopecia areata in comparison to those in patients with atopic dermatitis. Arch Dermatol Res 2007; 298: 397-401.

38. Skurkovich S, Korotky NG, Sharova NM, et al. Treatment of alopecia areata with anti-interferon-gamma antibodies. I Investig Dermatol Symp Proc 2005; 10: 283-4.

39. Korn T, Bettelli E, Oukka M, Kuchroo VK. IL-17 and Th17 cells. Annu Rev Immunol 2009; 27: 485-517.

40. Oukka M. Th17 cells in immunity and autoimmunity. Ann Rheum Dis 2008; 67 Suppl 3.: iii26-9.

41. Zhang L, Yuan S, Cheng G, Guo B. Type I IFN promotes IL10 production from $T$ cells to suppress Th17 cells and Th17associated autoimmune inflammation. PLoS One 2011; 6 : e28432.

42. Aytekin N, Akcali C, Pehlivan S. Investigation of interleukin-12, interleukin-17 and interleukin-23 receptor gene polymorphisms in alopecia areata. J Int Med Res 2015; 43: 526-34.

43. Atwa MA, Youssef N, Bayoumy NM, et al. T-helper 17 cytokines (interleukins 17, 21, 22, and 6, and tumor necrosis fac- 
tor-alpha) in patients with alopecia areata: association with clinical type and severity. Int J Dermatol 2016; 55: 666-72.

44. El-Morsy EH, Eid AA, Ghoneim H, et al. Serum level of interleukin-17A in patients with alopecia areata and its relationship to age. Int J Dermatol 2016; 55: 869-74.

45. Bilgic O, Sivrikaya A, Unlu A, et al. Serum cytokine and chemokine profiles in patients with alopecia areata. J Dermatolog Treat 2016; 27: 260-3.

46. Welsch K, Holstein J, Laurence A, et al. Targeting JAK/STAT signalling in inflammatory skin diseases with small molecule inhibitors. Eur J Immunol 2017; 47: 1096-107. 